\title{
Worldwide Prevalence of Intimate Partner Violence in Pregnancy. A Systematic Review and Meta-Analysis
}

\begin{abstract}
Rosario M. Román-Gálvez 1,2 , Sandra Martín-Peláez ${ }^{3,4 *}$, Borja M. Fernández-Félix ${ }^{5,6}$, Javier Zamora ${ }^{5,6}$, Khalid S. Khan ${ }^{3,6}$ and Aurora Bueno-Cavanillas ${ }^{3,4,6}$

${ }^{1}$ Departamento de Enfermería, Facultad de Ciencias de la Salud, Universidad de Granada, Granada, Spain, ${ }^{2}$ Unidad Asistencial Alhama de Granada, Servicio Andaluz de Salud, Granada, Spain, ${ }^{3}$ Departamento de Medicina Preventiva y Salud Pública, Facultad de Medicina, Universidad de Granada, Granada, Spain, ${ }^{4}$ Instituto de Investigación Biosanitaria de Granada IBS, Granada, Spain, ${ }^{5}$ Clinical Biostatistics Unit, Hospital Ramón y Cajal, Instituto Ramón y Cajal de Investigación Sanitaria (IRYCIS), Madrid, Spain, ${ }^{6}$ Centro de Investigación Biomédica en Red (CIBER) Epidemiología y Salud Pública, Instituto de Salud Carlos III, Madrid, Spain
\end{abstract}

OPEN ACCESS

Edited by:

MinJae Lee,

University of Texas Southwestern Medical Center, United States

Reviewed by:

Nigussie Tadesse Sharew, Hong Kong Polytechnic

University, China Susan M. Rees,

University of New South Wales, Australia

*Correspondence: Sandra Martín-Peláez sandramartin@ugr.es

Specialty section:

This article was submitted to Life-Course Epidemiology and Social Inequalities in Health, a section of the journal

Frontiers in Public Health

Received: 08 July 2021 Accepted: 09 August 2021 Published: 30 August 2021

Citation:

Román-Gálvez RM, Martín-Peláez S, Fernández-Félix BM, Zamora J,

Khan KS and Bueno-Cavanillas A

(2021) Worldwide Prevalence of Intimate Partner Violence in

Pregnancy. A Systematic Review and Meta-Analysis.

Front. Public Health 9:738459.

doi: 10.3389/fpubh.2021.738459
Background: Intimate partner violence (IPV) affects outcomes of mothers and their offspring. This systematic review collated the worldwide literature on the prevalence rates of different types of IPV in pregnancy.

Methods: Two reviewers independently identified cross sectional and cohort studies of IPV prevalence in pregnancy in online databases (PubMed, WOS and Scopus), selected and extracted data [participants' country, study quality, measurement tool (validation and purpose) and rates of IPV in pregnancy]. We considered a high quality study if it had a prospective design, an adequate sampling method, a sample size estimation, a response rate $>90 \%$, a contemporary ascertainment of IPV in the index pregnancy, and a well-developed detailed IPV tool. We performed random effects meta-analysis and explored reasons for heterogeneity of rates.

Results: One hundred fifty-five studies were included, of which 44 (28\%) met two-thirds of the quality criteria. Worldwide prevalence of physical (126 studies, 220,462 participants), psychological (113 studies, 189,630 participants) and sexual (98 studies, 155,324 participants) IPV in pregnancy was 9.2\% (95\% Cl 7.7-11.1\%, $\left.\right|^{2}$ 95.9\%), 18.7\% (15.1-22.9\%, I² 98.2\%), 5.5\% (4.0-7.5\%, $I^{2}$ 93.4\%), respectively. Where several types of IPV were reported combined, the prevalence of any kind of IPV (118 studies, 124,838 participants) was 25.0\% (20.3, 30.5\%, I² 98.6\%). IPV rates varied within and between continents, being the highest in Africa and the lowest in Europe $(p<0.001)$. Rates also varied according to measurement purpose, being higher for diagnosis than for screening, in physical $(p=0.022)$ and sexual $(p=0.014)$ IPV.

Conclusions: IPV prevalence in pregnancy varies across countries, with one-quarter of mothers exposed on average globally. Routine systematic antenatal detection should be applied worldwide.

Systematic Review Registration: identifier: CRD42020176131.

Keywords: pregnancy, intimate partner violence, domestic violence, prenatal care, prevalence 


\section{INTRODUCTION}

Intimate partner violence (IPV), defined as physical violence, psychological assault and sexual abuse (including coercive tactics) by a current or former intimate partner (1) is an avoidable global public health problem. IPV in pregnancy, despite being more common than other obstetric problems like preeclampsia or gestational diabetes (2) remains a neglected condition. It is associated with adverse pregnancy outcomes including increased risk of human immunodeficiency virus infection (3) perinatal depression (4) uterine rupture, hemorrhage, maternal death (5) prematurity, low birth weight, newborns small for gestational age (6) stillbirth (7) insufficient weight gain in pregnancy (8) and reduced levels of breastfeeding (9).

IPV prevalence in pregnancy is reported to vary according to the definition used (1) the measurement strategy $(10,11)$ and the socio-cultural context of the population studied $(2,12)$. These factors make comparison between individually reported rates difficult. An umbrella review of collated summaries of the published worldwide and country IPV prevalence data in pregnancy (13) showed that the existing evidence syntheses did not capture the totality of the disease burden, they did not always specify the gestational time point of IPV evaluation, and sometimes the IPV and domestic violence concepts were mixed up, leaving ambiguity. When assessed for quality, the existing reviews showed possible bias in study selection, general failure in addressing heterogeneity, and a narrow geographical coverage, focused on low-income countries (13). A robust updated systematic review of IPV prevalence in pregnancy is required to provide underpinning evidence for antenatal care policies.

Our objective was to synthesize worldwide prevalence data concerning physical, psychological, and sexual IPV in pregnancy and to explore reasons for variations in rates.

\section{METHODS}

The systematic review was carried out following prospective registration (PROSPERO ID: CRD42020176131) and reported according to the PRISMA statement (14).

\section{Literature Search and Selection}

Searches were conducted in PubMed, WOS and Scopus databases from inception to January 2021. The following search string combining medical subject headings $(\mathrm{MeSH})$ and free text terms was used ("'Intimate Partner Violence" AND ("Pregnancy" OR "Pregnant Women" OR "Prenatal Care")) AND "Prevalence"). In addition, relevant citations were scanning from reference lists of the selected articles. We contacted authors of key relevant citations by email for studies known to them of the subject. Two reviewers (RMRG and SMP) independently selected citation and studies meeting the following criteria: cross-sectional or cohort study design and a tool was used to estimate the rate of IPV in pregnancy. No language restrictions were applied.

\section{Data Extraction and Quality Assessment}

Two reviewers (RMR-G and SM-P) selected studies and extracted data independently, using a piloted form, to capture study, participant, and tool characteristics.

Adapting existing quality assessment tools $(15,16)$, we considered the following items and coding for evaluation of risk of bias: (a) a prospective design (yes, no, not reported or not evaluable); (b) a priori sample size estimation for precision of IPV rate (yes, no, not reported); (c) appropriate methods to capture a representative sample based on use of random or consecutive sampling, demonstration of similarity of sample characteristics to those of the population, (yes if any of these features were present, no, not reported or not evaluable); (d) contemporaneous ascertainment of IPV in the index pregnancy (yes, no, not reported); (e) use of a well-developed, detailed IPV tool with a published reference concerning its performance applied to the whole sample or to screen positive women, but not assessment by screening tools only [yes, no, not reported or not evaluable, using a published source (17); (f) relevance of IPV tool to local population either through development of the tool in their language or, if not originally developed in the same language, with translation and back translation, ideally with robust translated tool performance evaluations (yes, no, not reported or not evaluable); and (g) response rate over $90 \%$ (yes, no, not reported or not evaluable). We considered a study to be of high quality with respect to estimation of representative and unbiased IPV rates if it met at least 5 and low if met $<3$ of the criteria above. We calculated inter-reviewer agreement for data extraction concerning quality using kappa index to determine reliability (18). In cases of disagreement we used consensus and arbitration by a third reviewer $(\mathrm{ABC})$.

We extracted data concerning rates for each IPV type separately and combined. Numerator data concerning physical violence, psychological assault and sexual abuse were classified as reported by the authors of the selected papers.

\section{Data Synthesis}

Prevalence rates of IPV were estimated with $95 \%$ confidence intervals (CI) overall using a multilevel random effects logistic regression model with random intercepts for each study. Statistical heterogeneity was assessed graphically, using "forest" plots, and statistically, through the percentage of variation across studies (I squared statistic) and using the $\tau^{2}$ statistic. Sources of heterogeneity were evaluated by subgroups analysis, that were performed for geographical area (i.e., continent), type of instrument (validated, ad-hoc or unreported) and measurement purpose (screening, diagnosis or unreported). We used metaregression models to evaluate these associations as well as to assess the association with year of study publication. Funnel plots and Egger's tests were used to evaluate publication bias and small study effects, given extreme values of proportions (near 0 or 1) we used Freeman Tukey arcsin transformation to stabilize variances for this analysis (18-20). All statistical analyses were performed using Stata statistical software version $16.0(21)$. 


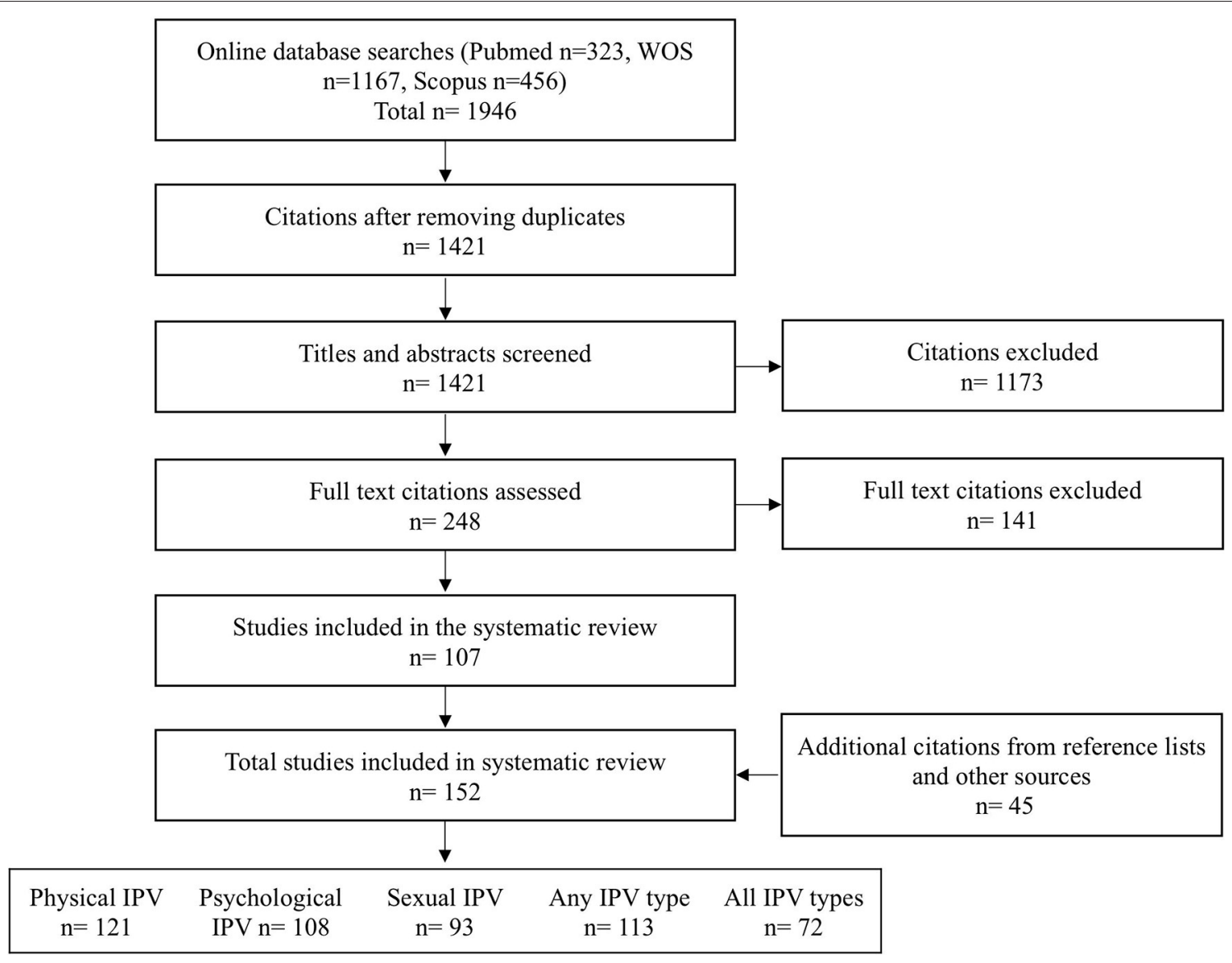

FIGURE 1 | Flowchart systematic review of intimate partner violence during pregnancy.

TABLE 1 | Meta-analysis by type of intimate partner violence (IPV) during pregnancy.

\begin{tabular}{|c|c|c|c|c|c|c|c|}
\hline IPV & Studies & $n$ & $N$ & Rate & $I^{2}(\%)$ & $P$-value heterog & $\mathrm{Tau}^{2}$ \\
\hline Psychological & 113 & 29,446 & 189,630 & $18.7(15.1,22.9)$ & 98.2 & 0.000 & 1.87 \\
\hline Any & 118 & 24,779 & 124,558 & $25.2(20.4,30.7)$ & 98.6 & 0.000 & 2.25 \\
\hline
\end{tabular}

$n=I P V$ events. $N=$ group size.

\section{RESULTS}

The electronic search yielded a total of 1,946 citations (Figure 1). After elimination of duplicates, 1,421 titles and abstracts were examined. Of these, 248 were found to be potentially relevant and their full articles were obtained. After reviewing these, 141 articles were excluded (Supplementary Table 1). The reference lists of the remaining 107 articles revealed 45 further citations. Finally, 152 articles (157 studies) met the inclusion criteria, providing data on 322,572 participants. Physical, psychological and sexual IPVs were all reported in $72(45.9 \%)$ studies; only one type of IPV, commonly physical, was reported in 32 (20.5\%) studies. The details of studies included in the systematic review on IPV prevalence in pregnancy are given in Supplementary Table 2. Study quality assessment (Supplementary Figure 1) revealed deficiencies in many areas of methodology, particularly those related to measurement tool, response rate and a priori sample size estimation. Overall, at least 5/7 quality criteria were met by $44(28.0 \%)$ studies, $3-4 / 7$ criteria by 92 (58.6\%) studies, and $<3 / 7$ criteria by $21(13.4 \%)$ studies. Inter-reviewer agreement concerning quality items was median Kappa 0.8 (range 0.6-1).

The data on IPV prevalence in pregnancy is summarized in Table 1. Worldwide prevalence of physical (126 studies, 220,462 participants), psychological (113 studies, 189,630 participants) and sexual (98 studies, 155,324 participants) IPV in pregnancy was $9.2 \%$ (95\% CI $7.7-11.1), 18.7 \%$ (95\% CI $15.1-22.9)$, 

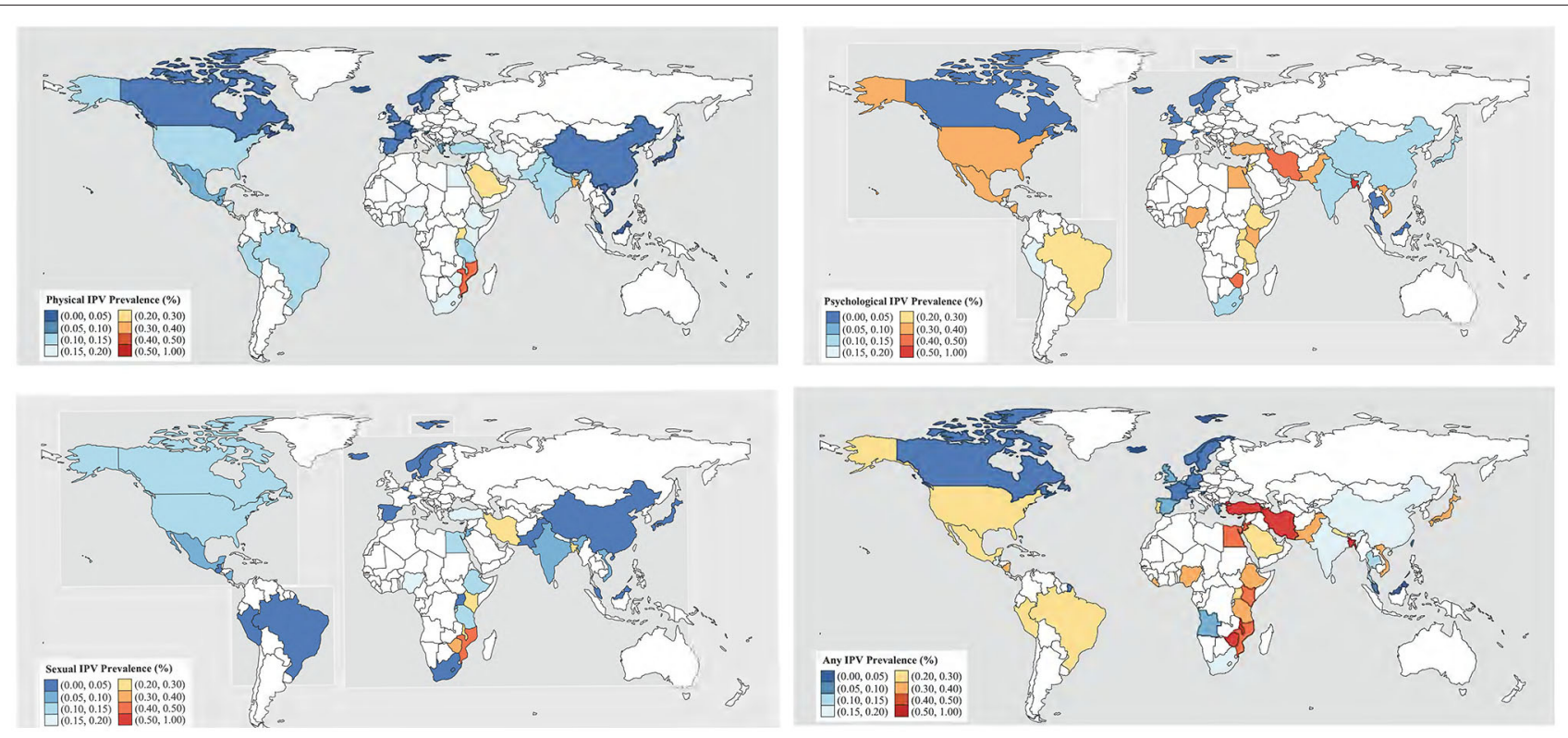

FIGURE 2 | World-wide distribution of intimate partner violence (IPV) types.

5.5\% (95\% CI 4.0-7.5), respectively. Where several types of IPV were reported combined, the prevalence of any kind of IPV (118 studies, 124,838 participants) was $25.0 \%$ (95\% CI 20.3-30.5). There was heterogeneity. The rates did not vary according to year of publication (Supplementary Figure 2). The retrieved data were from 52 countries. Figure 2 shows the worldwide prevalence rates by country concerning different types of IPV in pregnancy. Supplementary Table 3 gives the underlying data.

The prevalence rates of physical IPV in pregnancy ranged from $0.7 \%$ (22) to $55.1 \%$ (23) in 126 studies including 220,462 pregnant women. Worldwide prevalence rate of physical IPV in pregnancy was $9.2 \%$ (95\% CI 7.4-11.1). Prevalence figures were higher in Africa $(16.3 \%, 95 \%$ CI 13.5-19.6) than in Asia $(9.0 \%$, 95\% CI $6.5-12.3)$, North $(9.0 \%$, 95\% CI $5.3-$ 15.1) or South America (9.8\%, 95\% CI 7.3-13.0). There were only two studies from the Oceania region. The lowest prevalence was reported for Europe $(2.1 \%, 95 \%$ CI $1.3-$ 3.4). There were no differences according to the use or not of a validated tool, but prevalence figures were higher when the purpose of measurement was diagnosis instead of screening (Table 2).

The prevalence rates of psychological IPV in pregnancy ranged from $0.4 \%$ (24) to $79.8 \%$ (25) in 113 studies including 189,630 women. Global prevalence rate of psychological IPV in pregnancy was $(18.7 \%, 95 \%$ CI 15.15-22.9). Prevalence figures were higher in North América (28.6\%, 95\% CI 10.1-58.7) than in Africa $(25.2 \%, 95 \%$ CI $18.9-32.6)$, South America (23.4\%, 95\% CI 19.1-28.3) or Asia (19.3\%, 95\% CI 13.7-26.5). The lowest prevalence was reported for Europe $(4.2 \%, 95 \%$ CI 2.4-7.4). There were no differences according to the use or not of a validated tool, neither according to the purpose of measurement (Table 2).
The prevalence rates of sexual IPV in pregnancy ranged from $0.0 \%$ (26) to $45.6 \%$ (27) in 98 studies including 155,324 women. Global prevalence rate of sexual IPV in pregnancy was $(5.5 \%, 95 \%$ CI 40-7.5). Prevalence figures were higher in Africa (12.4\%, 95\% CI 8.6-17.4) than in North America (8.9\%, 95\% CI 3.8-19.4), Asia $(6.6 \%, 95 \%$ CI $4.1-10.7)$ or South America (2.7\%, 95\% CI 1.6-4.4). The lowest prevalence was reported for Europe (0.5\%, 95\% CI 0.3-0.9). There were no differences according to the use or not of a validated tool, but prevalence figures were higher when the purpose of measurement was diagnosis instead of screening (Table 2).

The prevalence rates of any type IPV in pregnancy ranged from $1.8 \%$ (28) to $99.5 \%$ (29) in 118 studies including 124,838 women. Global prevalence rate of any type IPV in pregnancy was $25.0 \%$ (95\% CI 20.4-30.7). Prevalence figures were higher in Africa $(36.1 \%, 95 \%$ CI $27.7-45.4)$ than in Asia $(32.1 \%$, 95\% CI 22.7-43.2), South $(25.6 \%, 95 \%$ CI 21.1-30.7) or North America (20.4\%, 95\% CI 6.9-47.1). The lowest prevalence was reported for Europe (5.1\%, 95\% CI 3.4-7.5). There were no differences according to the use or not of a validated tool, neither according to the scope of the study, in spite of that the prevalence rate was twice for diagnosis than for screening studies (Table 2).

For all IPVs we found a significant small studies effect (Supplementary Figure 3). Smaller studies show higher prevalence estimates.

\section{DISCUSSION}

In this evidence synthesis, IPV prevalence in pregnancy was found to be variable, with one-quarter of mothers exposed to violence. The retrieved data from over 50 countries, showed that overall one in ten mothers were exposed to physical IPV, one in 
TABLE 2 | Worldwide prevalence data for intimate partner violence (IPV) in pregnancy according to type of violence.

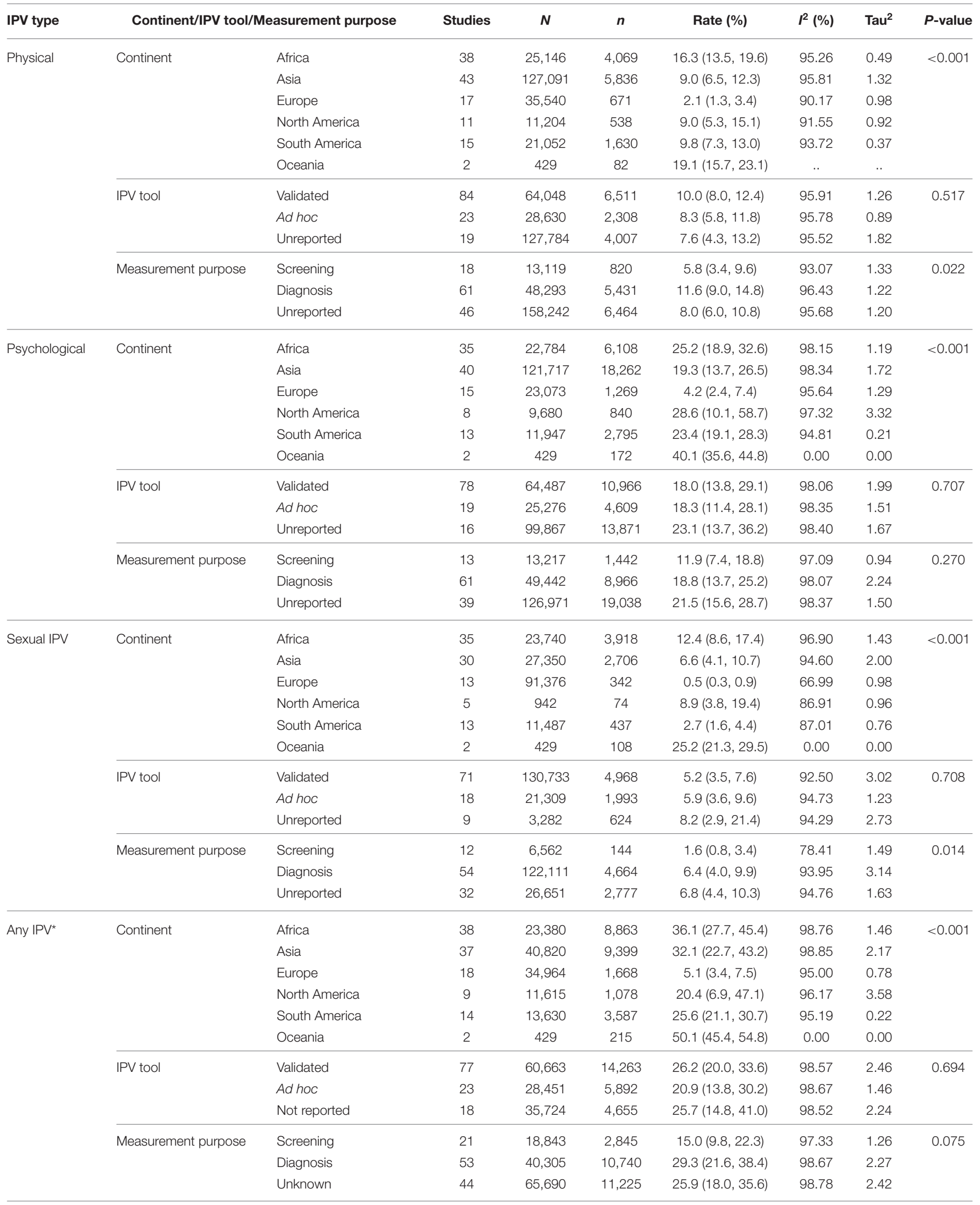

$n=I P V$ events. $N=$ group size.

${ }^{*}$ Any of the IPV types (physical, psychological or sexual) combined. 
five to psychological IPV and one in twenty to sexual IPV on average. IPV prevalence was heterogenous within and between continents. The geographical differences showed that IPV rates were the highest in Africa (except for psychological IPV which was higher in North America) and the lowest in Europe. In the East of Africa and Southeast of Asia the rates are twice or more as high as the rest. There were no reliable data from the Oceania region.

This review have strengths and some limitations. We conducted this review with a rigorous methodology and reported it transparently (14). A prospective review protocol was used, and an organized effort made to identify all the available evidence without any language restriction. Most of the include studies were published from 2013 onwards. The population demographics are unlikely to have undergone major changes over this period, making the findings relevant to the current time period. However, from the funnel plot analyses we should beware that publication bias may inflate the estimated prevalence of IPVs. We did not include studies from conflict areas and those that restricted to women in specific pregnancy risk groups, such as HIV infection or adolescents. Thus, our findings are representative of overall IPV rates among pregnancies that routinely book for antenatal care.

The primary studies included in the review were generally poor in methodological quality. For example, appropriate methods to capture a representative sample lacked among two in five studies and the response rate was below $90 \%$ among two in three studies. A well-developed, detailed IPV tool was used only by under a third of the included studies. By independently synthesizing each type of IPV we aimed to aggregate data within clearly defined categories. A random effects meta-analysis provided conservative estimates of precision, a feature particularly suitable when there is heterogeneity, as in our review. Both the quality and the rates of IPV varied among the included primary studies and we comprehensively explored the reasons for these variations. Our findings concerning different types of IPV are subject to correct classification by authors. For example, in psychological IPV, verbal and emotional violence were mixed together in some studies $(23,30-32)$ but not in others (33-35). We did not find any significant effect for the use of a validated measurement tool in explaining heterogeneity, but we did find that the geographical area and the measurement purpose (screening vs. diagnosis among studies of physical and sexual IPV) explained variation partially. Unexplained heterogeneity is a common unavoidable feature of meta-analyses. This review represents the best available evidence summary of the global estimates of IPV prevalence in pregnancy at the time of writing.

There is a large number of countries lacking information. We have included only prevalence studies, excluding data from administrative or general surveys, which generally provide lower prevalences (36-39) related to IPV in pregnancy. Recently the WHO reported that 1 in 3 women, around 736 million, are subjected to physical or sexual violence by an intimate partner or sexual violence from a non-partner (40). Our results confirm this information, adding concrete data regarding women's reproductive life period with respect to pregnancy. The information on the observed rates of IPV across pregnancy have implications for provision of health and social services. Policy makers need to focus on systematic screening with provision of improved security and social care to women at IPV risk.

Pregnancy provides a window for IPV detection, therefore routine antenatal screening should be the norm rather than the exception. Although the observed variations in geographical distribution may be related to the definitions used, study characteristics or study quality, the main reason could be the differences in socio-cultural acceptation of violence against women, and thus, IPV. Both, the normalization of IPV and its sociocultural stigmatization of women exposed to IPV make its detection difficult (41). Pregnancy could be used to inform women that violence should not be accepted (42). Since IPV tends to increase in frequency, intensity and impact from the moment that it appears, any solution for IPV depends on its early detection. Finding out the frequency of the different types of IPV and the global extent of this problem is the first step in its resolution.

Differences in prevalence found by different authors in the same country is not readily appreciated in the prevalence maps. For the same country, taking Ethiopia as an example, Gebrezgi et al., provided a prevalence of any type of IPV of $98 \%$ in the Tigrai (43) area, whereas Gebreslasie et al., showed a prevalence of $7.3 \%$ (44). In another example in Kenya, the $67 \%$ prevalence of any type of violence reported by Owaka et al. (45), was almost duplicate of the $37 \%$ rate provided by Makayoto et al. (46). In yet another example in Turkey Yanikkerem et al. (29) found prevalences higher than $90 \%$ in Manis, Cengiz, whereas prevalences were lower than $3 \%$ in Istanbul (47). Our prevalence maps make it possible to identify large geographic areas for which epidemiological data are not available, highlighting Russia, Kzajistan, Mongolia, all of North Africa, Australia, Argentina, Chile, Colombia, Venezuela, Bolivia, etc. Thus, there are significant gaps in the knowledge of the prevalence of IPV in pregnancy worldwide. Future research must pay close attention to the study design and to the use of validated measurement tools for validity and comparability of the results across studies and regions. Such efforts will need funding agencies to be willing to support broader and more systems oriented approach.

\section{CONCLUSION}

Overall, at least one in four pregnant women were exposed to one or another type of IPV in the worldwide literature. There were geographical differences. In the East of Africa and Southeast of Asia the figures are twice or more as high as the rest. Routine systematic IPV detection programs should introduced in antenatal care, a period of life when women at risk are likely to come in contact with health services. 


\section{DATA AVAILABILITY STATEMENT}

The raw data supporting the conclusions of this article will be made available by the authors, without undue reservation.

\section{AUTHOR CONTRIBUTIONS}

RMR-G and SM-P were responsible for searching and selecting reviews, data extraction, and reviewed and provided input into the manuscript. KSK and AB-C planned the study design and the analysis and coordinated the analysis and writing of the manuscript. BMF-F and JZ maintained the database and did the analysis. All authors contributed intellectually to the work.

\section{FUNDING}

KSK was a Distinguished Investigator funded by the Beatriz Galindo (senior modality) Program grant given to the University of Granada by the Spanish Ministry of Education.

\section{REFERENCES}

1. Dicola D, Spaar E. Intimate partner violence. Am Fam Physician. (2016) 94:646-51.

2. Devries KM, Kishor S, Johnson H, Stöckl H, Bacchus LJ, GarciaMoreno C, et al. Intimate partner violence during pregnancy: analysis of prevalence data from 19 countries. Reprod Health Matters. (2010) 18:15870. doi: 10.1016/S0968-8080(10)36533-5

3. World Health Organization. Global and regional estimates of violence against women: prevalence and health effects of intimate partner violence and nonpartner sexual violence. World Health Organization (2013). Available online at: https://apps.who.int/iris/handle/10665/85239

4. Halim N, Beard J, Mesic A, Patel A, Henderson D, Hibberd P. Intimate partner violence during pregnancy and perinatal mental disorders in low and lower middle income countries: a systematic review of literature, 1990-2017. Clin Psychol Rev. (2018) 66:117-35. doi: 10.1016/j.cpr.2017.11.004

5. El Kady D, Gilbert WM, Xing GB, Smith LH. Maternal and neonatal outcomes of assaults during pregnancy. Obstet Gynecol. (2005) 105:35763. doi: 10.1097/01.AOG.0000151109.46641.03

6. Donovan BM, Spracklen CN, Schweizer ML, Ryckman KK, Saftlas AF. Intimate partner violence during pregnancy and the risk for adverse infant outcomes: a systematic review and meta-analysis. BJOG. (2016) 123:128999. doi: 10.1111/1471-0528.13928

7. Pastor-Moreno G, Ruiz-Pérez I, Henares-Montiel J, Petrova D. Intimate partner violence during pregnancy and risk of fetal and neonatal death: a meta-analysis with socioeconomic context indicators. Am J Obstet Gynecol. (2020) 222:123-33.e5. doi: 10.1016/j.ajog.2019.07.045

8. Moraes CL, Amorim AR, Reichenheim ME. Gestational weight gain differentials in the presence of intimate partner violence. Int J Gynaecol Obstet. (2006) 95:254-60. doi: 10.1016/j.ijgo.2006.08.015

9. Lau Y, Chan KS. Influence of intimate partner violence during pregnancy and early postpartum depressive symptoms on breastfeeding among chinese women in Hong Kong. J Midwifery Womens Health. (2007) 52:e15e20. doi: 10.1016/j.jmwh.2006.09.001

10. Velasco C, Luna JD, Martin A, Caño A, Martin-de-Las-Heras S. Intimate partner violence against Spanish pregnant women: application of two screening instruments to assess prevalence and associated factors. Acta Obstet Gynecol Scand. (2014) 93:1050-8. doi: 10.1111/aogs.12459

11. Canterino JC, VanHorn LG, Harrigan JT, Ananth CV, Vintzileos AM. Domestic abuse in pregnancy: a comparison of a self-completed domestic abuse questionnaire with a directed interview. Am J Obstet Gynecol. (1999) 181:1049-51. doi: 10.1016/S0002-9378(99)70079-7

\section{ACKNOWLEDGMENTS}

The authors are grateful to Ms. Purificacion CamposGallego for her contribution as a patient and public representative.

\section{PATIENT AND PUBLIC INVOLVEMENT}

A patient representative was involved throughout the review, participated in review management meetings, and contributed to the evaluation of topic importance, interpretation of results and the generation of recommendations.

\section{SUPPLEMENTARY MATERIAL}

The Supplementary Material for this article can be found online at: https://www.frontiersin.org/articles/10.3389/fpubh. 2021.738459/full\#supplementary-material

12. Garcia-Moreno C, Jansen HA, Ellsberg M, Heise L, Watts CH. Prevalence of intimate partner violence: findings from the WHO multi-country study on women's health and domestic violence. Lancet. (2006) 368:12609. doi: 10.1016/S0140-6736(06)69523-8

13. Roman-Galvez RM, Martín-Peláez S, Martinez-Galiano JM, Khan KS, Bueno-Cavanillas A. Prevalence of intimate partner violence in pregnancy: an umbrella review. Int J Environ Res Public Health. (2021) 18:707 doi: 10.3390/ijerph18020707

14. Liberati A, Altman DG, Tetzlaff J, Mulrow C, Gøtzsche PC, Ioannidis JPA, et al. The PRISMA statement for reporting systematic reviews and metaanalyses of studies that evaluate healthcare interventions: explanation and elaboration. BMJ. (2009) 339:b2700 doi: 10.1136/bmj.b2700

15. Latthe P, Latthe M, Say L, Gülmezoglu M, Khan KS. WHO systematic review of prevalence of chronic pelvic pain: a neglected reproductive health morbidity. BMC Public Health. (2006) 6:177. doi: 10.1186/1471-2458-6-177

16. Pretlove SJ, Radley S, Toozs-Hobson PM, Thompson PJ, Coomarasamy A, Khan KS. Prevalence of anal incontinence according to age and gender: a systematic review and meta-regression analysis. Int Urogynecol J Pelvic Floor Dysfunct. (2021) 17:407-17. doi: 10.1007/s00192-005-0014-5

17. Escuela Andaluza de Salud Pública. Catálogo de instrumentos para cribado y frecuencia del maltrato físico psicológico y sexual. Granada: Escuela Andaluza de Salud Pública (2021).

18. Landis JR, Koch GG. The measurement of observer agreement for categorical data. Biometrics. (1977) 33:159. doi: 10.2307/2529310

19. Hunter JP, Saratzis A, Sutton AJ, Boucher RH, Sayers RD, MJ B. In metaanalyses of proportion studies, funnel plots were found to be an inaccurate method of assessing publication bias. J Clin Epidemiol. (2014) 67:897903. doi: 10.1016/j.jclinepi.2014.03.003

20. Freeman MF, Tukey JW. Transformations related to the angular and the square root. Ann Math Statist. (1950) 21:607-11.

21. Stata Corp. 2019. Stata Statistical Software: RRelease 16. College Station, TX: StataCorp LLC (2019).

22. Topbas M, Uensal M, Can G, Bacak A, Oezguen S. The effect of pregnancy on the physical and sexual abuse of women that presented to a state hospital in Trabzon, Turkey. Turk J Med Sci. (2008) 38:335-42.

23. Idoko P, Ogbe E, Jallow O, Ocheke A. Burden of intimate partner violence in The Gambia - a cross sectional study of pregnant women. Reprod Health. (2015) 21:12-34. doi: 10.1186/s12978-015-0023-x

24. Finnbogadóttir H, Dykes A-K, Wann-Hansson C. Prevalence of domestic violence during pregnancy and related risk factors: a crosssectional study in southern Sweden. BMC Womens Health. (2014) 14:63. doi: 10.1186/1472-6874-14-63 
25. Bailey BA. Partner violence during pregnancy: prevalence, effects, screening, and management. Int J Womens Health. (2010) 2:183-97. doi: 10.2147/ijwh.s8632

26. Lukasse M, Schroll A-M, Ryding EL, Campbell J, Karro H, Kristjansdottir $\mathrm{H}$, et al. Prevalence of emotional, physical and sexual abuse among pregnant women in six European countries. Acta Obstet Gynecol Scand. (2014) 93:66977. doi: 10.1111/aogs.12392

27. Chaquisse E, Fraga S, Meireles P, Macassa G, Soares J, Mbofana F, et al. Sexual and physical intimate partner violence among women using antenatal care in Nampula, Mozambique. J Public Health Afr. (2018) 9:16. doi: 10.4081/jphia.2018.744

28. Maciel MNA, Blondel B, Saurel-Cubizolles MJ. Physical violence during pregnancy in france: frequency and impact on the health of expectant mothers and new-borns. Matern Child Health J. (2019) 23:1108-16. doi: 10.1007/s10995-019-02747-y

29. Yanikkerem E, Karadas G, Adiguzel B, Sevil U. Domestic violence during pregnancy in turkey and responsibility of prenatal healthcare providers. Am J Perinatol. (2006) 23:93-103. doi: 10.1055/s-2006-931802

30. Komori K, Komori M, Eitoku M, Muchanga SMJ, Ninomiya H, Kobayashi T, et al. Verbal abuse during pregnancy increases frequency of newborn hearing screening referral: The Japan Environment and Children's Study. Child Abuse Negl. (2019) 90:193-201. doi: 10.1016/j.chiabu.2019.01.025

31. Coonrod DV, Bay RC, Mills TE, Gamble SL. Asymptomatic bacteriuria and intimate partner violence in pregnant women. Am J Obstet Gynecol. (2007) 196:581.e1-.e4. doi: 10.1016/j.ajog.2007.03.018

32. Gurkan OC, Eksi Z, Deniz D, Circir H. The influence of intimate partner violence on pregnancy symptoms. J Interpers Violence. (2020) 35:52341. doi: $10.1177 / 0886260518789902$

33. Mammadov B, Vural G, Tosun O. Risk factors associated with intimate partner violence during pregnancy in Northern Cyprus. Cyprus J Med Sci. (2018) 3:9-14. doi: 10.5152/cjms.2018.312

34. Oweis A, Gharaibeh M, Alhourani R. Prevalence of violence during pregnancy: findings from a Jordanian survey. Matern Child Health J. (2010) 14:437-45. doi: 10.1007/s10995-009-0465-2

35. Iliyasu Z, Abubakar IS, Galadanci HS, Hayatu Z, Aliyu MH. Prevalence and risk factors for domestic violence among pregnant women in Northern Nigeria. J Interpers Violence. (2013) 28:868-83. doi: 10.1177/0886260512455872

36. Alhusen JL, Geller R, Jellig J, Budhathoki C, Decker M. Intimate partner violence, small for gestational age birth and cigarette smoking in the pregnancy risk assessment monitoring system. J Womens Health. (2002) 2018) 27:458-65. doi: 10.1089/jwh.2017.6322

37. Charles P, Perreira KM. Intimate partner violence during pregnancy and 1-year post-partum. J Fam Violence. (2007) 22:609-19. doi: 10.1007/s10896-007-9112-0

38. Durand JG, Schraiber LB. Violence during pregnancy among public health care users in the Greater São Paulo area: prevalence and associated factors. Revista Brasileira de Epidemiologia. (2007) 10:310-22. doi: 10.1590/S1415-790X2007000300003

39. Leone JM, Lane SD, Koumans EH, DeMott K, Wojtowycz MA, Jensen J, et al. Effects of intimate partner violence on pregnancy trauma and placental abruption. J Womens Health. (2010) 19:1501-9. doi: 10.1089/jwh.2009.1716
40. WHO. Violence Against Women Prevalence Estimates 2018. Global, Regional and National Prevalence Estimates for Intimate Partner Violence Against Women and Global and Regional Prevalence Estimates for Non-partner Sexual Violence Against Women. 2021 ed. Geneva: WHO (2021).

41. Kennedy AC, Prock KA. "I still feel like I am not normal": a review of the role of stigma and stigmatization among female survivors of child sexual abuse, sexual assault, and intimate partner violence. Trauma Violence Abuse. (2018) 9:512-27. doi: 10.1177/1524838016673601

42. Chaves K, Eastwood J, Ogbo FA, Hendry A, Jalaludin B, Khanlari $S$, et al. Intimate partner violence identified through routine antenatal screening and maternal and perinatal health outcomes. BMC Pregnancy Childbirth. (2019) 19:357. doi: 10.1186/s12884-0192527-9

43. Gebrezgi BH, Badi MB, Cherkose EA, Weldehaweria NB. Factors associated with intimate partner physical violence among women attending antenatal care in Shire Endaselassie town, Tigray, northern Ethiopia: a cross-sectional study, July 2015. Reprod Health. (2017) 14:76. doi: 10.1186/s12978-01 7-0337-y

44. Gebreslasie KZ, Weldemariam S, Gebre G, Mehari MA. Intimate partner violence during pregnancy and risk of still birth in hospitals of Tigray region Ethiopia. Italian J Pediatr. (2020) 46:107. doi: 10.1186/s13052-020-0 0857-w

45. Owaka IO, Nyanchoka MK, Atieli HE. Intimate partner violence in pregnancy among antenatal attendees at health facilities in West Pokot county, Kenya. Pan Afr Med J. (2017) 28:229. doi: 10.11604/pamj.2017.28.22 9.8840

46. Makayoto LA, Omolo J, Kamweya AM, Harder VS, Mutai J. Prevalence and associated factors of intimate partner violence among pregnant women attending Kisumu District Hospital, Kenya. Matern Child Health J. (2013) 17:441-7. doi: 10.1007/s10995-012-1015-x

47. Cengiz H. Domestic violence against pregnant women: a prospective study in a metropolitan city, Istanbul. J Turk Ger Gynecol Assoc. (2014) 15:207 doi: 10.5152/jtgga.2014.65632

Conflict of Interest: The authors declare that the research was conducted in the absence of any commercial or financial relationships that could be construed as a potential conflict of interest.

Publisher's Note: All claims expressed in this article are solely those of the authors and do not necessarily represent those of their affiliated organizations, or those of the publisher, the editors and the reviewers. Any product that may be evaluated in this article, or claim that may be made by its manufacturer, is not guaranteed or endorsed by the publisher.

Copyright (C) 2021 Román-Gálvez, Martín-Peláez, Fernández-Félix, Zamora, Khan and Bueno-Cavanillas. This is an open-access article distributed under the terms of the Creative Commons Attribution License (CC BY). The use, distribution or reproduction in other forums is permitted, provided the original author(s) and the copyright owner(s) are credited and that the original publication in this journal is cited, in accordance with accepted academic practice. No use, distribution or reproduction is permitted which does not comply with these terms. 